\title{
Proposal to elevate Mycobacterium avium complex ITS sequevar MAC-Q to Mycobacterium vulneris
} sp. nov.

\author{
Correspondence \\ J. van Ingen \\ jakko.van.ingen@rivm.nl
}

\author{
J. van Ingen, ${ }^{1,2}$ M. J. Boeree, ${ }^{1}$ K. Kösters, ${ }^{3,4}$ A. Wieland, ${ }^{5}$ E. Tortoli, ${ }^{6}$ \\ P. N. R. Dekhuijzen ${ }^{1}$ and D. van Soolingen ${ }^{2}$
}

The Mycobacterium avium complex (MAC) consists of four recognized species, Mycobacterium avium, Mycobacterium colombiense, Mycobacterium intracellulare and Mycobacterium chimaera, and a variety of other strains that may be members of undescribed taxa. We report on two isolates of a scotochromogenic, slowly growing, non-tuberculous Mycobacterium species within the $M$. avium complex from a lymph node and an infected wound after a dogbite of separate patients in The Netherlands. The extrapulmonary infections in immunocompetent patients suggested a high level of virulence. These isolates were characterized by a unique nucleotide sequence in the $16 \mathrm{~S}$ rRNA gene, $99 \%$ similar to Mycobacterium colombiense, and the MAC-Q 16S-23S internal transcribed spacer (ITS) sequence. Sequence analyses of the $h s p 65$ gene revealed $97 \%$ similarity to $M$. avium. The $r p o B$ gene sequence was $98 \%$ similar to $M$. colombiense. Phenotypically, the scotochromogenicity, positive semi-quantitative catalase and heat-stable catalase tests, negative tellurite reductase and urease tests and susceptibility to hydroxylamine and oleic acid set these isolates apart from related species. High-performance liquid chromatography analysis of cell-wall mycolic acid content revealed a unique pattern, related to that of M. avium and M. colombiense. Together, these findings supported a separate species status within the Mycobacterium avium complex. We propose elevation of scotochromogenic M. avium complex strains sharing this $16 \mathrm{~S}$ gene and MAC-O ITS sequence to separate species status, for which the name Mycobacterium vulneris sp. nov. is proposed. The type strain is

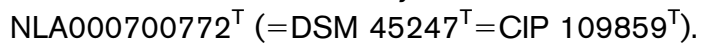

Abbreviations: ITS, internal transcribed spacer; MAC, Mycobacterium avium complex; MIC, minimal inhibitory concentration.

The GenBank/EMBL/DDBJ accession numbers for the $16 \mathrm{~S}$ rRNA and rpoB gene sequences of strain NLA000700772 ${ }^{\top}$ are EU834055 and EU834057, respectively. The accession numbers for the hsp65 gene sequences of strains NLA000700772 ${ }^{\top}$ and NLA009601918 are EU834054 and EU834056, respectively.

Phylogenetic trees based on $h s p 65$ and $r p o B$ gene sequences of selected Mycobacterium species are available as supplementary material with the online version of this paper.
Mycobacterium avium complex (MAC) bacteria are the most frequently isolated non-tuberculous mycobacteria worldwide and are capable of causing a wide spectrum of clinical disease. Pulmonary disease, mostly in patients with pre-existent pulmonary diseases, is most common, followed by lymphadenitis in immunocompetent children and disseminated disease in systemically immunocompromised patients (Griffith et al., 2007).

The taxonomy of the MAC has been a subject of debate for decades. The MAC was long divided into two species, $M$. avium and Mycobacterium intracellulare, and a number of 
unnamed bacteria not belonging to these two taxa. Frothingham and Wilson noted that sequencing of the 16S-23S internal transcribed spacer (ITS) revealed a wide range of genetic diversity among reference strains of those unnamed MAC bacteria, suggesting the presence of several as yet undefined species (Frothingham \& Wilson, 1993). Two such groups have recently been elevated to species rank, Mycobacterium chimaera and Mycobacterium colombiense (Tortoli et al., 2004; Murcia et al., 2006). In this study, we report on a novel $M$. avium complex member, related to $M$. colombiense, which was isolated from two patients in the Netherlands.

A previously healthy, 42-year-old woman presented with a painful wound in her left lower leg 7 weeks after a dogbite. Several small white lesions with limited ulceration were noted. Surgical wound excision and oral amoxicillin and clavulanic acid had limited success. An abscess with fistula to the skin and spontaneous wound rupture prompted renewed surgical debridement, Mycobacterium culture, and vacuum therapy. The cultures yielded a non-tuberculous Mycobacterium. A third wound debridement and vacuum therapy eventually led to symptomatic improvement and wound closure. Mycobacterium cultures from samples of the third debridement remained negative.

A previously healthy, two-year-old girl presented at another hospital with painless swelling of a right cervical lymph node and violaceous overlying skin. No other symptoms were reported. A biopsy revealed granulomatous inflammation, but no acid-fast bacilli were visible on direct microscopy. Cultures of the biopsy material yielded a nontuberculous Mycobacterium and surgery was successfully performed. No relapse has been noted since.

Both isolates were sent to the National Institute for Public Health and the Environment (RIVM), Bilthoven, The Netherlands. The RIVM is the national mycobacteria reference laboratory, which provides identification, typing and drug susceptibility testing.
We investigated the biochemical and phenotypical features and performed high-performance liquid chromatography (HPLC) analysis of cell-wall mycolic acid content, using previously described procedures (Kent \& Kubica, 1985; CDC, 1996). We used the HPLC mycobacterium library (available online at http://www.MycobacToscana.it) for visual comparisons.

For primary identification, we used the Inno-LiPA Mycobacteria v2 (Innogenetics, Ghent, Belgium) and Hain GenoType CM/AS (Hain Lifesciences, Nehren, Germany) reverse line-blots. To identify the isolates to the species level we sequenced the complete 16S rRNA gene, the 16S-23S ITS region and partial hsp65 and rpoB genes, using previously published primers and methods (Springer et al., 1996; Roth et al., 1998; Telenti et al., 1993; Adékambi et al., 2003). The sequences obtained were compared with the GenBank/EMBL/DDBJ (National Center for Biotechnology Information: http:// www.ncbi.nlm.nih.gov) sequence database.

The 16S rRNA gene sequences of the Mycobacterium isolates of the two cases were aligned with those of reference strains in the MAC using CLUSTAL_X software (Thompson et al., 1997). The resulting topology and tree, inferred by using neighbour-joining and visualized using the MEGA 4.0 software package (Tamura et al., 2007), were evaluated by bootstrap analyses based on 1000 resamplings (Fig. 1). The tree was rooted with M. tuberculosis $\mathrm{H} 37 \mathrm{Rv}^{\mathrm{T}}$ as an outgroup.

We tested the presence of the IS1245 element by amplification of a 427 bp internal fragment, using P1 and P2 primers, as previously described (Guerrero et al., 1995).

Drug susceptibility testing was performed using the 25-well agar dilution method (van Klingeren et al., 2007). Isoniazid, rifampicin, rifabutin, ethambutol, clarithromycin, ciprofloxacin, cycloserine, prothionamide, amikacin, clofazimine and streptomycin were included in the test panel.

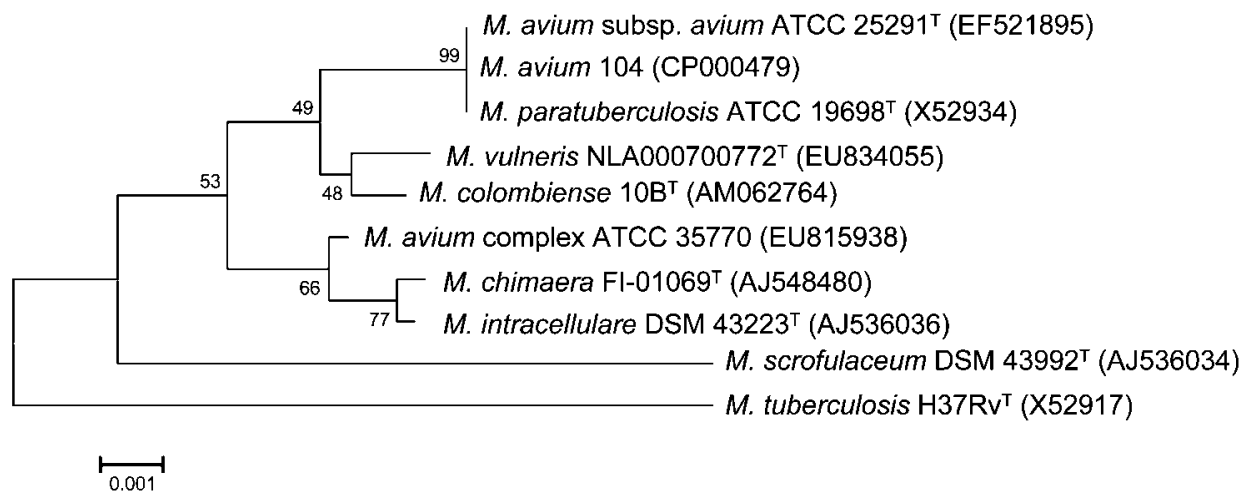

Fig. 1. Phylogenetic relationships of the type strain of M. vulneris sp. nov. and related species of Mycobacterium, based on 16S rRNA gene sequences. The neighbour-joining tree was constructed based on bootstrap analysis with 1000 resamplings and visualized with MEGA 4.0 (Tamura et al., 2007). Percentage bootstrap values are given at nodes. Bar, 0.001 substitutions per nucleotide. 
On Middlebrook 7H10, Ogawa and Stonebrink media (Oxoid; The Netherlands), the bacteria produce film-like growth with small, smooth, bright yellow-pigmented colonies after 3 weeks incubation at $36{ }^{\circ} \mathrm{C}$. Growth on Middlebrook $7 \mathrm{H} 10$ agar was only observed at 24,30 and $36{ }^{\circ} \mathrm{C}$. Optimal growth occurred at $36{ }^{\circ} \mathrm{C}$. Colony morphology on Middlebrook 7H10 agar was similar at all temperatures.

The Inno-LiPA Mycobacteria v2 assay revealed only reaction with the MAIS (M. avium-intracellulare-scrofulaceum) complex probe, thus identifying the isolates as $M$. avium complex members, different from $M$. avium, $M$. intracellulare and M. scrofulaceum. Analysis using the Hain GenoType CM assay (which uses the 23S rRNA gene as its target) incorrectly identified the strain as $M$. intracellulare. This may reflect $23 \mathrm{~S}$ gene sequence similarity of our isolates with $M$. intracellulare. Currently available commercial identification kits for non-tuberculous mycobacteria do not have sufficient discriminative power to recognize particular subgroupings among the MAC isolates. This may suffice in the clinical setting, as the treatment of MAC disease, to date, is independent of exact speciation results (Griffith et al., 2007). However, an improved recognition of clinically relevant subgroupings within the complex may improve clinical management and eventually support research on the epidemiology and pathogenesis of MAC disease.

Sequencing of the complete $16 \mathrm{~S}$ rRNA genes of the two isolates revealed a unique sequence most closely related to MAC bacteria (Table 1). The multisequence alignment results of the $16 \mathrm{~S}$ rRNA gene sequence clarified its taxonomical position within the MAC as being most closely related to M. colombiense (Fig. 1). The 16S-23S ITS sequences of both strains were identical to the previously described MAC-Q ITS sequevar (GenBank no. AF315833); it differed in $6 \mathrm{bp}$ from the M. colombiense ITS sequevar
(Table 1). The previously published MAC-Q strain (Mijs et al., 2002) was that isolated from patient two, the girl with lymphadenitis. We performed multisequence alignment of all published MAC ITS sequevars currently available in GenBank (Fig. 2). Additional sequencing of the $h s p 65$ and $r p o B$ genes revealed unique sequences, detailed in Table 1. Again, sequences were most similar to MAC members and related to $M$. avium rather than $M$. intracellulare (Table 1). Both strains had unique hsp65 sequences, the difference being a $\mathrm{G} \longrightarrow \mathrm{A}$ substitution at position 140 (corresponding to codon 538 of the $M$. tuberculosis $\mathrm{H}_{37 \mathrm{Rv}^{\mathrm{T}}} h s p 65$ gene). The $r p o B$ sequence indicated that the isolates were related specifically to $M$. colombiense, in line with the 16S rRNA gene results (Table 1). We aligned the $r p o B$ and $h s p 65$ sequences with those of related Mycobacterium species, using the same methods as for the 16S rRNA gene sequence. In addition, concatenated $16 \mathrm{~S}$ rRNA, hsp65 and rpoB gene sequences were aligned with concatenated sequences of the related Mycobacterium species (Stackebrandt et al., 2002). The resulting topologies and trees are available as supplementary material in IJSEM Online (Supplementary Figs S1, S2 and S3).

We were able to demonstrate, by PCR, the presence of the IS1245 element in the genomes of the two isolates (results not shown). This supported its taxonomic position within the MAC, related to $M$. avium rather than $M$. intracellulare, although a minority of the $M$. intracellulare and other MAC strains are known to harbour this element (Mijs et al., 2002).

Phenotypic identification revealed a pattern generally similar to MAC strains, with negative tests for niacin accumulation, nitrate reduction, $\beta$-glucosidase, Tween 80 hydrolysis, 3 day arylsulfatase, urease and growth on MacConkey agar, but positive for $68{ }^{\circ} \mathrm{C}$ catalase. Our isolates were divergent in their positive semi-quantitave catalase test, susceptibility to hydroxylamine and oleic acid,

Table 1. Sequence comparison results for M. vulneris NLA000700772 ${ }^{\top}$

\begin{tabular}{|c|c|}
\hline Target & Results of GenBank/EMBL/DDBJ comparison \\
\hline \multirow[t]{4}{*}{ 16S rRNA (1471 bp) GenBank EU834055 } & $99 \%$ M. colombiense $10 \mathrm{~B}^{\mathrm{T}}(1436 / 1439 \mathrm{bp})$ \\
\hline & $99 \%$ M. chimaera FI-0169 $(1432 / 1439$ bp $)$ \\
\hline & $99 \%$ M. avium 104 (1463/1471 bp) \\
\hline & $99 \%$ M. paratuberculosis ATCC $19698^{\mathrm{T}}(1463 / 1471 \mathrm{bp})$ \\
\hline \multirow[t]{4}{*}{ 16S-23S ITS (281 bp) GenBank AF315833 MAC-Q } & $99 \%$ MAC-R, AF315834 (279/281 bp) \\
\hline & $98 \%$ MAC-E (ATCC 35847), L07852 (278/281 bp) \\
\hline & 98 \% MAC-F, L07853 (277/281 bp) \\
\hline & $97 \%$ M. colombiense $10 \mathrm{~B}^{\mathrm{T}}(275 / 281 \mathrm{bp})$ \\
\hline \multirow[t]{4}{*}{ rpoB (726 bp) GenBank EU834057 } & $98 \%$ M. colombiense CIP $108962^{\mathrm{T}}(690 / 701 \mathrm{bp})$ \\
\hline & $95 \%$ M. chimaera DSM $44623^{\mathrm{T}}(669 / 701 \mathrm{bp})$ \\
\hline & $94 \%$ M. avium 104 (678/714 bp) \\
\hline & $94 \%$ M. avium ATCC $25291^{\mathrm{T}}(665 / 701 \mathrm{bp})$ \\
\hline \multirow[t]{4}{*}{ hsp65 (424 bp) GenBank EU834054 } & $97 \%$ M. avium ATCC $25291^{\mathrm{T}}(415 / 424 \mathrm{bp})$ \\
\hline & $97 \%$ M. avium 104 (415/424 bp) \\
\hline & $97 \%$ M chimaera CIP $107892^{\mathrm{T}}(414 / 424 \mathrm{bp})$ \\
\hline & $97 \%$ M. intracellulare ATCC $13950^{\mathrm{T}}(412 / 424 \mathrm{bp})$ \\
\hline
\end{tabular}




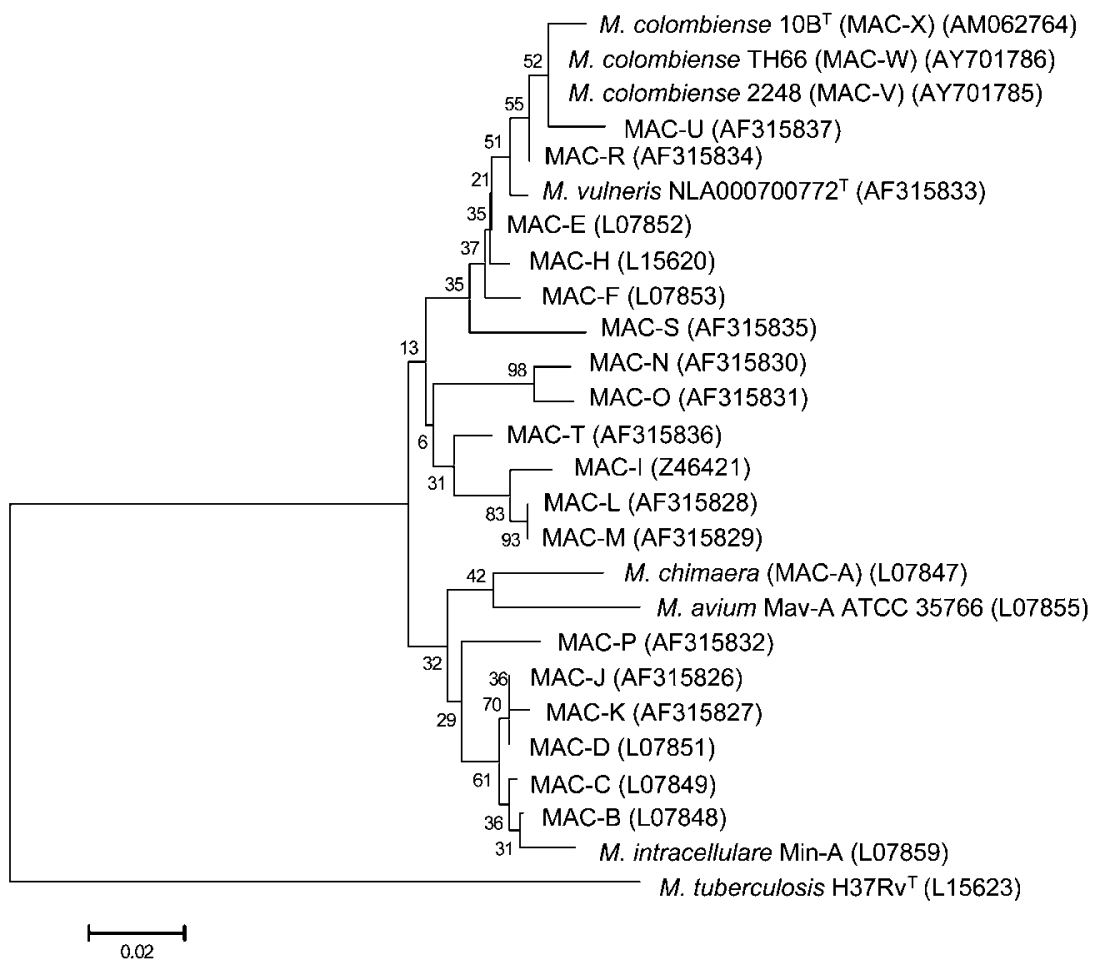

Fig. 2. Phylogenetic tree based on ITS sequences showing the relationships of $M$. chimaera and other sequevars of MAC. Each organism is indicated by the sequevar name and GenBank accession number. The neighbour-joining tree was constructed based on bootstrap analysis with 1000 resamplings and visualized with MEGA 4.0 (Tamura et al., 2007). Percentage bootstrap values are given at nodes. Bar, 0.02 substitutions per nucleotide.

Table 2. Biochemical identification results

Taxa: 1, M. vulneris sp. nov.; 2, M. colombiense; 3, M. avium; 4, M. intracellulare; 5, M. chimaera. All species grow at $25^{\circ} \mathrm{C}$. All species do not accumulate niacin, reduce nitrate, hydrolyse Tween 80 or grow on MacConkey agar. The growth rate of all species in Middlebrook $7 \mathrm{H} 10$ medium at $36{ }^{\circ} \mathrm{C}$ is slow. + , Positive; - , negative; $+/-$, variable; ND, not determined.

\begin{tabular}{|c|c|c|c|c|c|}
\hline Test item & 1 & 2 & 3 & 4 & 5 \\
\hline Catalase $>45 \mathrm{~mm}$ & + & + & - & - & - \\
\hline Tellurite reduction & - & $\mathrm{ND}$ & + & $+1-$ & $+1-$ \\
\hline 3-day Arylsulfatase & - & $+1-$ & - & - & - \\
\hline Urease & - & + & - & - & - \\
\hline Colony morphology & Smooth & Rough & Smooth & Smooth & Smooth \\
\hline Growth at $42{ }^{\circ} \mathrm{C}$ & - & - & $+1-$ & $+1-$ & - \\
\hline \multicolumn{6}{|l|}{ Tolerance to: } \\
\hline $\mathrm{TCH} \dagger$ & + & ND & + & + & + \\
\hline Thiacetazone $\left(10 \mu \mathrm{g} \mathrm{ml}^{-1}\right)$ & + & ND & + & + & + \\
\hline Isoniazid $\left(500 \mu \mathrm{g} \mathrm{ml}^{-1}\right) \ddagger$ & + & ND & + & + & + \\
\hline
\end{tabular}

${ }^{*}$ NC, Non-chromogenic; SC, scotochromogenic.

$\dagger$ Thiophen-2-carboxylic acid hydrazide.

$\ddagger$ On Middlebrook 7H10 agar. 
as well as their scotochromogenicity. Positive semiquantitative catalase tests were also noted for M. colombiense, which differs from our isolates in its negative urease test and colony morphology and pigmentation (Table 2).

The HPLC pattern of the isolate comprises 3 clusters of peaks; the first cluster was the main one and included four major peaks. The second and third emerged later and were close to each other, presenting four and three peaks, respectively (Fig. 3). This pattern was consistent among most strains included in the MAC, although the $M$. colombiense pattern is characterized by increasing peak heights within the first cluster and the second cluster is absent in M. chimaera (Tortoli et al., 2004; Murcia et al., 2006). The relative heights of the peaks in the second and third cluster varied within the MAC. M. avium mostly presents lower peaks in the third cluster, as for the $M$. vulneris isolates. M. colombiense usually presents lower peaks in the second cluster (Fig. 3).

Applying the 25-well agar dilution method for drug susceptibility testing (van Klingeren et al., 2007), we recorded resistance to isoniazid ( $\mathrm{MIC}>20 \mu \mathrm{g} \mathrm{ml} \mathrm{m}^{-1}$ ),

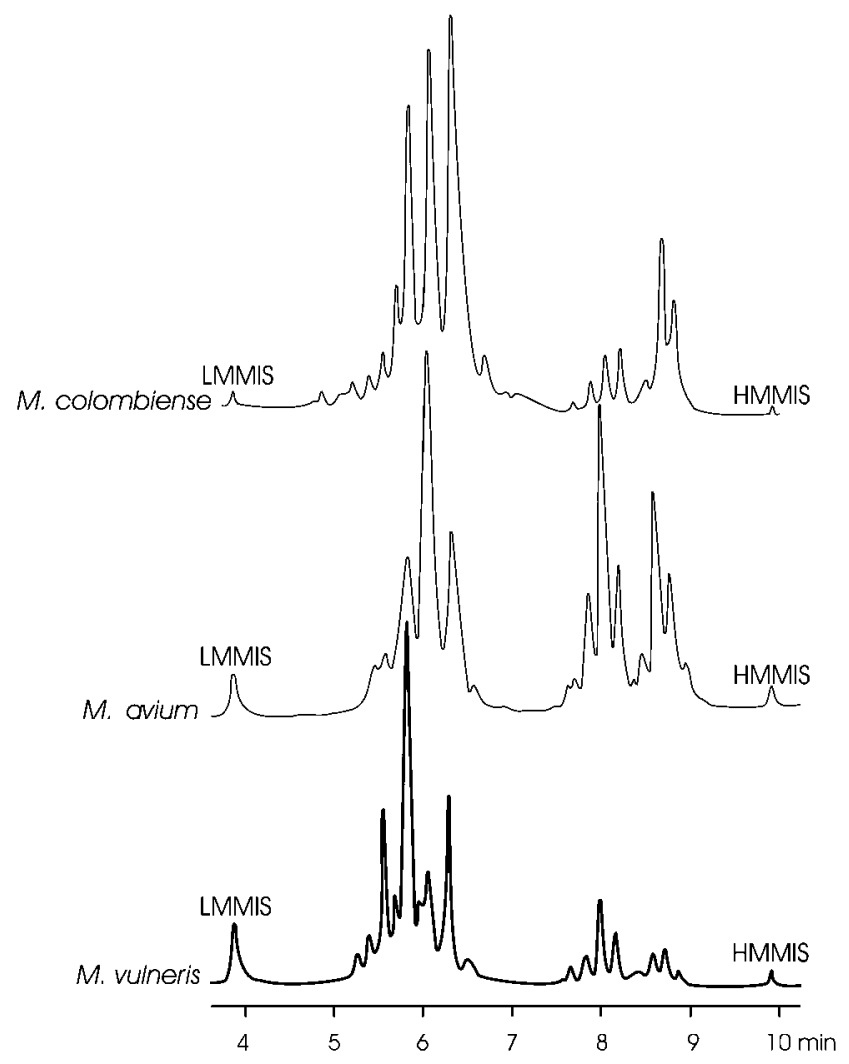

Fig. 3. Mycolic acid patterns of $M$. vulneris, $M$. avium and $M$. colombiense obtained by HPLC analysis. The M. vulneris pattern is similar to that of other M. avium complex members, but does not share the increasing peak heights within the first cluster, typical for M. colombiense. HMMIS, high molecular mass internal standard; LMMIS, low molecular mass internal standard. ethambutol (MIC $20 \mu \mathrm{g} \mathrm{ml} \mathrm{ml}^{-1}$ ), streptomycin (MIC $>20 \mu \mathrm{g} \mathrm{ml}^{-1}$ ), amikacin (MIC $20 \mu \mathrm{g} \mathrm{ml}^{-1}$ ) and ciprofloxacin (MIC $>16 \mu \mathrm{g} \mathrm{ml} \mathrm{m}^{-1}$ ), with susceptibility to rifampicin (MIC $1 \mu \mathrm{g} \mathrm{ml}^{-1}$ ), rifabutin (MIC $1 \mu \mathrm{g} \mathrm{ml} \mathrm{m}^{-1}$ ), clarithromycin (MIC $4 \mu \mathrm{g} \mathrm{ml} \mathrm{m}^{-1}$ ), clofazimine (MIC= $<0.5 \mu \mathrm{g} \mathrm{ml}{ }^{-1}$ ), cycloserine (MIC $20 \mu \mathrm{g} \mathrm{ml}^{-1}$ ) and prothionamide (MIC $2 \mu \mathrm{g} \mathrm{ml}^{-1}$ ).

Previous authors have suggested the presence of multiple species within MAC, based on various phenotypic and genetic traits (Frothingham \& Wilson, 1993; Wayne et al., 1996; Wayne \& Sramek, 1992). Based on the phenotypic and genotypic features reported above, we believe that our isolates represent one such species. The extrapulmonary infections in immunocompetent patients suggest a high level of virulence.

\section{Description of Mycobacterium vulneris sp. nov.}

Mycobacterium vulneris [vul'ne.ris; L. gen. n. vulneris of a wound (vulnus), from which the type strain was isolated].

Stains acid-alcohol-fast. Cells are short rods, with occasional coccoid forms. No cording, spores or filaments are present. On Middlebrook 7H10, Ogawa and Stonebrink media, mature growth develops after 28 days incubation at $36{ }^{\circ} \mathrm{C}$; growth is slower at 25 and $30{ }^{\circ} \mathrm{C}$ and no growth occurs at $42{ }^{\circ} \mathrm{C}$. Colonies are small, scotochromogenic and bright yellow in appearance. Negative for niacin accumulation, nitrate reduction, $\beta$-glucosidase, Tween 80 hydrolysis, tellurite reduction, 3 day arylsulfatase, urease and growth on MacConkey agar, but positive for $68{ }^{\circ} \mathrm{C}$ catalase and semi-quantitative catalase. Tolerant to isoniazid, thiophen-2-carboxylic acid hydrazide, $p$-nitrobenzoic acid and thiacetazone, but not to oleic acid or hydroxylamine. Readily identifiable by its unique HPLC pattern and 16S rRNA, $h s p 65$ and $r p o B$ gene sequences. The 16S-23S ITS region was previously described as the MACQ ITS sequevar.

The type strain, NLA000700772 ${ }^{\mathrm{T}}\left(=\mathrm{DSM} 45247^{\mathrm{T}}=\mathrm{CIP}\right.$ $109859^{\mathrm{T}}$ ), was isolated from a wound debridement specimen.

\section{References}

Adékambi, T., Colson, P. \& Drancourt, M. (2003). rpoB-based identification of nonpigmented and late-pigmenting rapidly growing mycobacteria. J Clin Microbiol 41, 5699-5708.

CDC (1996). Standardized Method for HPLC Identification of Mycobacteria. Atlanta, USA: Department of Health and Human Services, Centers for Disease Control and Prevention.

Frothingham, R. \& Wilson, K. H. (1993). Sequence-based differentiation of strains in the Mycobacterium avium complex. J Bacteriol 175, 2818-2825.

Griffith, D. E., Aksamit, T., Brown-Elliot, B. A., Catanzaro, A., Daley, C., Gordin, F., Holland, S. M., Horsburgh, R., Huitt, G. \& other authors (2007). An official ATS/IDSA statement: diagnosis, treatment, and prevention of nontuberculous mycobacterial diseases. Am J Respir Crit Care Med 175, 367-416. 
Guerrero, C., Bernasconi, C., Burki, D., Bodmer, T. \& Telenti, A. (1995). A novel insertion element from Mycobacterium avium, IS1245, is a specific target for analysis of strain relatedness. J Clin Microbiol 33, 304-307.

Kent, P. T. \& Kubica, G. P. (1985). Public Health Mycobacteriology. A Guide For The Level III Laboratory. Atlanta, USA: Department of Health and Human Services.

Mijs, W., de Haas, P., Rossau, R., van der Laan, T., Rigouts, L., Portaels, F. \& van Soolingen, D. (2002). Molecular evidence to support a proposal to reserve the designation Mycobacterium avium subsp. avium for bird-type isolates and ' $M$. avium subsp. hominissuis' for the human/porcine type of M. avium. Int J Syst Evol Microbiol 52, $1505-1518$.

Murcia, M. I., Tortoli, E., Menendez, M. C., Palenque, E. \& Garcia, M. J. (2006). Mycobacterium colombiense sp. nov., a novel member of the Mycobacterium avium complex and description of MAC-X as a new ITS genetic variant. Int J Syst Evol Microbiol 56, 2049-2054.

Roth, A., Fischer, M., Hamid, M. E., Michalke, S., Ludwig, W. \& Mauch, H. (1998). Differentiation of phylogenetically related slowly growing mycobacteria based on 16S-23S rRNA gene internal transcribed spacer sequences. J Clin Microbiol 36, 139-147.

Springer, B., Stockman, L., Teschner, K., Roberts, G. D. \& Böttger, E. C. (1996). Two-laboratory collaborative study on identification of mycobacteria: molecular versus phenotypic methods. J Clin Microbiol 34, 296-303.

Stackebrandt, E., Frederiksen, W., Garrity, G. M., Grimont, P. A., Kämpfer, P., Maiden, M. C., Nesme, X., Rosselló-Mora, R., Swings, J. \& other authors (2002). Report of the ad hoc committee for the reevaluation of the species definition in bacteriology. Int J Syst Evol Microbiol 52, 1043-1047.
Tamura, K., Dudley, J., Nei, M. \& Kumar, S. (2007). MEGA4: Molecular Evolutionary Genetics Analysis (MEGA) software version 4.0. Mol Biol Evol 24, 1596-1599.

Telenti, A., Marchesi, F., Balz, M., Bally, F., Böttger, E. C. \& Bodmer, T. (1993). Rapid identification of mycobacteria to the species level by polymerase chain reaction and restriction enzyme analysis. J Clin Microbiol 31, 175-178.

Thompson, J. D., Gibson, T. J., Plewniak, F., Jeanmougin, F. \& Higgins, D. G. (1997). The CLUSTAL_X windows interface: flexible strategies for multiple sequence alignment aided by quality analysis tools. Nucleic Acids Res 25, 4876-4882.

Tortoli, E., Rindi, L., Garcia, M. J., Chiaradonna, P., Dei, R., Garzelli, C., Kroppenstedt, R. M., Lari, N., Mattei, R. \& other authors (2004). Proposal to elevate the genetic variant MAC-A, included in the Mycobacterium avium complex, to species rank as Mycobacterium chimaera sp. nov. Int J Syst Evol Microbiol 54, 1277-1285.

van Klingeren, B., Dessens-Kroon, M., van der Laan, T., Kremer, K. \& van Soolingen, D. (2007). Drug susceptibility testing of Mycobacterium tuberculosis complex using a high throughput, reproducible, absolute concentration method. J Clin Microbiol 45, 2662-2668.

Wayne, L. G. \& Sramek, H. A. (1992). Agents of newly recognized or infrequently encountered mycobacterial diseases. Clin Microbiol Rev $5,1-25$.

Wayne, L. G., Good, R. C., Böttger, E. C., Butler, R., Dorsch, M., Ezaki, T., Gross, W., Jonas, V., Kilburn, J. \& other authors (1996). Semantide- and chemotaxonomy-based analyses of some problematic phenotypic clusters of slowly growing mycobacteria, a cooperative study of the International Working Group on Mycobacterial Taxonomy. Int J Syst Bacteriol 46, 280-297. 